\title{
CONSUMO PATRIMONIAL: ENTRE EL MERCADO Y LA CULTURA
}

\author{
HERITAGE CONSUMPTION: BETWEEN MARKET AND CULTURE
}

\author{
Macarena Hernández-Ramírez y Esteban Ruiz-Ballesteros ${ }^{1}$
}

\begin{abstract}
El protagonismo del patrimonio en la sociedad contemporánea resulta indiscutible. Su concurrencia es capital en temáticas aparentemente tan dispares como la convivencia multicultural o el desarrollo económico. Nuestro objetivo en este artículo es mostrar la oportunidad de integrar la perspectiva del consumo en el estudio del patrimonio. Partimos de una consideración dinámica y abierta de lo patrimonial que se centra en los procesos de patrimonialización antes que en la naturaleza de los objetos patrimoniales; en el concurso de los sujetos en esos procesos antes que en el carácter normativo, estético o político de los bienes patrimoniales; y en el efecto cultural del patrimonio antes que en su papel como reflejo de la cultura. Se propone un acercamiento teórico al consumo patrimonial desde la experiencia proporcionada por dos casos de estudio etnográfico muy distintos en su naturaleza y casuística -Agua Blanca (Ecuador) y Riotinto (España)-.
\end{abstract}

Palabras claves: patrimonio, consumo, mercado y cultura.

The role of heritage in contemporary society is indisputable. Its relevance is crucial in seemingly disparate topics such as multiculturalism or economic development. Our goal in this article is to show the opportunity of integrating the perspective of consumption into the study of heritage. We start from a dynamic and open consideration of heritage that focuses on processes rather than on the nature of heritage objects; on the contribution of the items subject to those processes rather than the regulatory, aesthetic or political nature of the patrimonial assets; and on the cultural impact of heritage rather than its role as a reflection of culture. We propose a theoretical approach to heritage consumption from the experience provided by two very different ethnographic case-studies in their nature and particularities: Agua Blanca (Ecuador) and Riotinto (Spain).

Key words: Heritage, consumption, market, culture.

La afirmación de que el patrimonio es fruto de un proceso de construcción socio-técnico-política ya no supone ninguna novedad, sin embargo conviene profundizar en su naturaleza procesual. El patrimonio no solo es producto de un proceso, sino que es un proceso en sí mismo, de ahí que no podamos considerarlo en ningún momento un producto acabado o estático.

Desde esta perspectiva conviene preguntarse -más allá de cuál o cómo sea el elemento sujeto a la acción patrimonial- por el efecto y alcance que pueden llegar a tener los procesos patrimonializadores. El objetivo no es otro que desvelar las posibles y variables relaciones que se establecen entre los sujetos sociales y los objetos patrimoniales. Somos conscientes que patrimonializar no es precisamente una actividad discreta, neutra o aséptica, sino todo lo contrario. Y que por tanto precisa ser estudiada sin renunciar a su inherente complejidad multidimensional, y a su carácter dinámico y conflictivo.
La patrimonialización obedece a la interacción de distintos agentes sociales que propician un conjunto de intervenciones con las que se significa especialmente un elemento o conjunto de elementos (tangibles e intangibles), lo que implica en muchas ocasiones un proceso de resemantización de los mismos. Se genera así un discurso desde el que describir y habitar el mundo, una suerte de sacralización laica de la realidad. Esta manera de mirar el patrimonio nos lo presentaría como una causa, además de como una consecuencia. Es en el devenir de estos procesos de patrimonialización donde se establece un campo discursivo y de práctica (si es que ambos pudieran separarse), desde el que poder aprehender de manera compleja la concurrencia de distintas propuestas de representación y praxis de la realidad.

Evidentemente, así entendida, el interés de la patrimonialización no se centra en los elementos patrimoniales, sino en el propio hecho social de convertir parte de la realidad en patrimonio (Arrieta

1 Departamento de Antropología Social, Psicología Básica y Salud Pública. Universidad Pablo de Olavide, Sevilla, España. Ctra de Utrera Km. 1 Sevilla 4013. mherram@upo.es; eruibal@upo.es 
2009; Hernández-Ramírez y Ruiz-Ballesteros 2006; Pereiro 2003; Prat 2006; Quintero 2009) y sobre todo, en vivirla como tal. Esta mirada acerca de lo patrimonial cambia completamente la perspectiva: de su naturaleza construida se da el salto a sus usos sociales (Alsayyad 2001; Ariño 2002; Cruces 1998; García Canclini 1995; Hernández-Ramírez y Ruiz-Ballesteros 2005, 2008; Quintero 2005; Rosas 1999). De ahí que nuestras preguntas también se transformen: de "¿qué es patrimonio?" pasamos a cuestionarnos ¿qué hace la gente con el patrimonio?, ¿qué hace el patrimonio con la gente? Se cruzan así perspectivas tanto culturalistas como economicistas, y en medio de todo ello aparece el consumo (García Canclíni 1995) como dimensión prioritaria del patrimonio, evidenciando su más radical naturaleza procesual. Un consumo polifónico, que a veces y paradójicamente, es el gran antídoto de una producción patrimonial con voluntad hegemónica. De este modo, entendemos, se ofrece una perspectiva complementaria y necesaria a su vez, en la que se presenta al patrimonio como acto de consumo (individual y/o colectivo) en el que emergen las distintas maneras de comunicación-relación con él y se hace explícito su carácter procesual, nunca acabado: el patrimonio se conforma realmente en tanto se consume.

Con los dos casos de estudio que presentamos en este artículo (Agua Blanca y Riotinto) buscamos no solo conocer la forma en que determinados comportamientos, objetos o ideas se convierten en patrimonio, sino cómo se hace el patrimonio y sobre todo qué hace el patrimonio. Con ello incidimos en que el patrimonio no es solo una lectura, sino sobre todo una práctica del mundo que habitamos. Para ello ilustraremos desde la etnografía las relaciones entre patrimonio y consumo.

\section{Patrimonialización en Agua Blanca y Riotinto}

Tras varias décadas trabajando con un enfoque antropológico respecto de patrimonio y su turistización en América Latina y España, hemos seleccionado dos casos significativos para ilustrar en qué medida y cómo el patrimonio es objeto de consumo. En Riotinto (España) -lugar emblemático para la patrimonialización de la mineríacomenzamos nuestras investigaciones a principios de los noventa; pero de manera más focalizada en los procesos patrimoniales desarrollamos una primera campaña de trabajo de campo intensivo en 2003 (seis meses) y posteriormente otra entre 2007-2009 (seis meses). En Agua Blanca (Ecuador) -una relevante experiencia de patrimonialización natural y arqueológica- se ha desarrollado trabajo de campo intensivo entre 2006 y 2008 (seis meses), retomándose la investigación de campo en 2016 (un mes). En ambos casos hemos conseguido desarrollar una perspectiva longitudinal suficiente para captar el devenir de la patrimonialización. El material etnográfico que aquí se utiliza es fruto de este periodo de investigación antropológica, centrado fundamentalmente en los agentes y actores de los procesos de patrimonialización (poblaciones locales, técnicos, visitantes, etc.), así como en los propios contextos de consumo patrimonial (museos, visitas, rituales, etc.). Para ello hemos utilizado distintas técnicas habituales en etnografía, centradas principalmente en fuentes primarias: observación participante, entrevistas en profundidad, grupos focales y microbiografías.

\section{Agua Blanca}

Agua Blanca es una pequeña comunidad ecuatoriana que sin llegar a los 300 habitantes ocupa una extensión aproximada de 9.000 ha en el corazón del Parque Nacional Machalilla (Manabí, Ecuador). Este territorio es relevante por su ecosistema de bosque seco tropical y por los abundantes restos arqueológicos de la cultura manteña. Hasta los años setenta del siglo veinte la comunidad fue completamente dependiente de una hacienda y posteriormente sus actividades giraron en torno a la caza, la tala de madera, la producción de carbón vegetal y la venta ilegal de restos arqueológicos. En 1979 se creó el Parque Nacional y con él, a fin de una mejor conservación ambiental, la intención de deportar a la población a otra zona del país. Se inicia entonces un proceso que convierte en patrimonio distintos elementos clave del entorno que ocupaba la comunidad, tanto desde una perspectiva cultural como natural.

En Agua Blanca, el propio entorno pasó a ser declarado patrimonio en virtud de sus valores naturales. Convertir el entorno en patrimonio significó resignificarlo como naturaleza, construir un nuevo objeto, invocar nuevas palabras, otras maneras de nombrar. Donde los aguablanquenses hablaban del "valle", la "sabana" y la "montaña", surgieron desde el parque y el lenguaje científico 
el "bosque seco tropical", el "bosque de garúa". Con esas denominaciones llegaron prohibiciones y persecuciones para acabar con el régimen extractivo que marcaba la economía local: las especies que cazaban y los árboles que talaban se convirtieron de la noche a la mañana en elementos especialmente protegidos, lo que eran prácticas de subsistencia pasaron a ser tipificadas como actividades delictivas. Se imponía así el imperativo científico-conservacionista que esgrimía un discurso que representaba el entorno como un lugar de especial protección ambiental, en gran medida incompatible con la presencia humana. Entre 1979 y 1985 se vivió el fragor de una imposición patrimonial en extremo conflictiva. Convertir al entorno en naturaleza y a esta en patrimonio conlleva, en principio, un extrañamiento radical para los habitantes de la comunidad respecto de su espacio vital (RuizBallesteros 2009).

Desde una perspectiva cultural, a partir de mediados de los ochenta los abundantes restos arqueológicos de la cultura manteña que salpicaban el territorio de la comunidad fueron sometidos a estudio científico, registrados y convertidos en patrimonio. Hasta ese momento los aguablanquenses solían vender los restos que afloraban a la superficie a los huaqueros como una forma complementaria de obtener recursos económicos. Esas piedras y cerámicas no tenían más valor para ellos. Fueron las excavaciones, desarrolladas en distintas campañas e implementadas desde presupuestos de arqueología social y participativa, las que propiciaron la implicación directa y continuada de gran número de habitantes locales, al punto que muchos de ellos aprendieron la lógica del trabajo arqueológico, y alcanzaron una visión amplia del poblamiento histórico del territorio (McEwan et al. 2006). En este caso la conversión de las ruinas y los objetos en patrimonio no fue conflictivo sino más bien al contrario. La arqueología fue una fuente de empleo y de conocimiento, y finalmente, con la construcción de un museo local y de una ruta de visita (similar a lo acontecido en otros lugares del continente americano, ver Martínez y Oconitrillo 2012), constituyó un patrimonio susceptible de explotación turística que, sumado a su oferta como patrimonio natural, constituye hoy la principal fuente de ingreso de la comunidad (Ruiz-Ballesteros 2009; Ruiz-Ballesteros y Gual 2012, Ruiz-Ballesteros y Hernández-Ramírez 2011). Paradójicamente los problemas con el parque y la conservación ambiental terminaron cuando se puso en marcha una oferta de turismo comunitario que tenía al patrimonio cultural y natural como objeto de explotación turística.

\section{Riotinto}

$\mathrm{Al}$ contrario de lo que sucede en buena parte del territorio americano (Angelo 2014), es lugar común en Europa el desmantelamiento de las zonas minero-industriales. Enclaves que un día fueron hervidero de la actividad extractiva a gran escala son hoy presas del abandono y el olvido. Muchas minas han sido rehabilitadas nuevamente, en este caso como enclaves patrimoniales, procurando atraer un turismo cultural que aúna ocio y conocimiento. Los territorios mineros de Andalucía no se han sustraído de este tipo de procesos (Hernández-Ramírez y Ruiz-Ballesteros 2005 y 2006; Ruiz-Ballesteros 1999a y 2001a; Ruiz-Ballesteros y HernándezRamírez 2007, 2008). En ellos, lo que otrora fueran instalaciones industriales y zonas de extracción de mineral, hoy son escenarios para representar la cultura y el paisaje minero.

Riotinto (Andalucía, España) es una comarca emblemática de la minería mundial. Los vestigios de la explotación minera se remontan a varios milenios, alcanzando su máximo esplendor durante el Imperio Romano y la propiedad británica de las minas (1873-1954). Las minas se cerraron tras numerosas vicisitudes a principios del siglo XXI, dejando una comarca que pierde población desde entonces, sumida en una profunda crisis socioeconómica y una reapertura de la mina que aún está sujeta a incertidumbre. La desactivación minera se inició en la década de 1980 y muy pronto se acompañó de una apuesta patrimonial que cristalizó en la creación de la Fundación Riotinto (1987), la conformación progresiva de un Parque Minero de vocación turística y la declaración de la comarca (a partir de sus elementos minero-industriales) como bien de interés cultural por parte del gobierno de Andalucía en 2005. Habiendo estudiado en profundidad todo este proceso patrimonializador (Iglesias y Ruiz 1999; Romero et al. 2003; Ruiz-Ballesteros 1999b) nos interesó finalmente la forma en que los últimos mineros conceptualizaban y percibían las propias instalaciones y paisajes en los que habían trabajado, ahora convertidos en patrimonio y sujetos a la mirada de los turistas.

En Riotinto tanto la desactivación de la minería como el proceso patrimonializador reproduce 
estrategias ya utilizadas en otras muchas zonas minero-industriales europeas, por tanto desactivación y patrimonialización no pueden entenderse más que como una expresión de la globalización. Pero esta constatación, por más que acertada, no nos informa de toda la extensión que el proceso patrimonializador pudiera tener. Nos explica qué se ha convertido en patrimonio, quién lo ha hecho, incluso cómo se ha hecho; pero no qué ha hecho el patrimonio con la gente o la gente con el patrimonio; en definitiva: qué ha supuesto para la población local todo este proceso. Igualmente podríamos decir para el caso de Agua Blanca: la patrimonialización del entorno natural o de restos arqueológicos son lugares comunes a nivel mundial. Y nosotros nos preguntamos ¿dónde queda la gente?

\section{El patrimonio y su gente en Agua Blanca y Riotinto}

En Agua Blanca convertir el entorno natural y cultural en patrimonio ha supuesto un cambio profundo para la población. Cultura y naturaleza tienen una dimensión ontológica, epistemológica y política que se sustancia en el objeto patrimonial en sí. Cómo se asumen estos conceptos y cómo se lidia con ellos es básico para comprender las prácticas que componen la cotidianidad en Agua Blanca. Transformar en patrimonio el entorno cotidiano constituye un proceso complejo que funde apropiación y mercantilización, de la mano del patrimonio y del turismo (Hernández-Ramírez y Ruiz-Ballesteros 2011). Convertir en patrimonio distintos elementos del medio en el que viven los aguablanquenses ha tenido una múltiple significación: (1) la transformación radical en la forma de ver y situarse ante el medio, (2) la reafirmación territorial de la comunidad tras asumir plenamente el proceso de patrimonialización; (3) el cambio radical en la forma de vida y sustento (con el nacimiento del turismo como principal actividad local); y (4) la emergencia de un proceso identitario en forma de etnogénesis que tiene como eje a los restos arqueológicos (HernándezRamírez y Ruiz-Ballesteros 2011). Así, lo interesante del caso de Agua Blanca no es tanto el patrimonio constituido en sí mismo sino la manera en que este se ha conformado y sobre todo la relación práctica que los habitantes de la comunidad mantienen con él: dinamizador económico, elemento legitimador, marcador identitario. El caso de Agua Blanca evidencia esa complejidad y carácter procesual del patrimonio, pues el patrimonio en esta comunidad no está cerrado, sino que sigue haciéndose día a día en un contexto de permanente tensión con el mercado y el Estado en cuanto a la protección y el uso de los elementos que constituyen el patrimonio natural y cultural local. El protagonismo de los aguablanquenses es evidente: en el fondo son ellos los principales consumidores de su patrimonio.

En el caso de Riotinto la patrimonialización tiene al menos una doble intencionalidad. Por un lado trata de mantener la memoria de los trabajos y actividades minero-industriales, actuando sobre la identidad. Por otro, procura ser uno de los vectores de regeneración socioeconómica de las comarcas mineras por medio del turismo. Ambas estrategias buscan mitigar la depresión demográfica, social y económica en la que inevitablemente caen estas zonas tras el desmantelamiento de las actividades que le dieron razón de ser y se encuadran entre las políticas de rehabilitación promovidas a nivel local, regional, nacional y europeo. Pero ¿qué efecto tiene esta patrimonialización sobre las personas más directamente implicadas? ¿En qué medida afecta a su forma de representarse en el entorno y relacionarse con él? Efectivamente, sin un abordaje de estas cuestiones no se completa una visión adecuada de los procesos de patrimonialización, que no acaban precisamente con la "producción del patrimonio".

Para nosotros atender a los sujetos ha supuesto un clarificador punto de partida a la hora de comprender comportamientos, pensamientos y relaciones de la gente con/en el patrimonio. Los mineros que sintieron cómo la minería se demonizaba durante todo el proceso de desactivación y se estigmatizaba por sus consecuencias ambientales (Ruiz-Ballesteros 2001b), han resemantizado la actividad minera y su paisaje, provocando una doble rehabilitación. Por un lado, ese paisaje antes objeto de vergüenza por su impacto sobre el espacio "natural" se lee como un distintivo de la comarca y se reclama abiertamente su "naturaleza geológica" a modo de una naturalización de la mina (Ruiz et al. 2009). Por otro lado, el sindicalismo y el trabajo minero que marcaron sociopolíticamente a la comarca y que asimismo han experimentado una erosión de sus bases y principios, se ven también restituidos por la patrimonialización que supone una revalorización de los mineros y su cultura. Todo ello resultaría un tanto opaco si no se evidenciara en los sujetos del proceso y en las nuevas relaciones que estos establecen con los distintos objetos patrimonializados, sean las 
cortas, los malacates, el ferrocarril o el propio río Tinto (Ruiz y Rubio 2008).

allí siento el fin de la mina. Es ya un lugar de recuerdo, de máquinas que no se ven, de trazos de polvo y humo, que sólo existen en mi memoria. Un grandioso vacío. Sobre esa Corta soy capaz de ver las perforadoras, los tractores, las excavadoras, los volquetes arriba y abajo (...) y nosotros ahí trabajando con sol y con lluvia (...) los compañeros y nuestras luchas (...). Nada de todo eso hay ya...

Algunas veces llegan turistas. Ya sé que ellos no ven el silencio que yo veo, ni las ausencias; ellos vienen a ver el patrimonio. Me gusta poner la oreja, escuchar sus comentarios. En alguna ocasión me entrometo para responder a algunas de sus inquietudes. Me sorprende lo que ellos miran y lo que ven, lo que les llama la atención y sus comentarios. La mayoría no aciertan a comprender cómo se hizo aquello ni para qué, pero parece que no les hace falta. Se admiran de todo. Incluso el agua que inunda la corta se convierte en un motivo de admiración. Esa agua no es más que la evidencia del fin de la mina (...). Los turistas lo ven todo con otros ojos. Eso debe ser el patrimonio ¿no? Miran lo mismo que yo pero lo ven de otra manera. Es curioso. No alcanzan a ver lo que fue, ni lo imaginan, pero se admiran de manera extraordinaria de lo que hay. Sus comentarios se paran en detalles sin importancia... Y aún así se admiran, les gusta, se impresionan por la grandiosidad del lugar (...) Y eso lo hemos hecho nosotros, los mineros, y ellos vienen a verlo (...) antes no podíamos imaginar que resultara interesante para nadie. iQuién nos lo iba a decir! ... (notas en diario de campo, entrevista informal con minero pre-jubilado, 2008).

Estos mineros denotan "desorientación" y "deriva" tras la desactivación de la actividad minera y el patrimonio parece ser un ancla para ellos, ya que les procura tanto una reincorporación a la mina (ahora objeto turístico), como asimismo un elemento de reactivación y reconocimiento identitario. Esto es también parte del proceso de patrimonialización que nunca acaba, y que supone el encuentro de miradas, de sujetos, de rehabilitación de una comarca y de sus habitantes. La conformación del patrimonio no reside principalmente en sus atributos objetivos, sino ante todo en los ojos que miran y las palabras que de ahí surgen; en definitiva en sus sujetos.

Por eso, la primera mañana que compartimos con un grupo de visitantes el recorrido por el museo minero de Riotinto, fuimos conscientes de la necesidad de analizarlos en profundidad y cualitativamente. El perfil de visitantes (individuales, escolares, universidades, agencias de viajes, otros) que se establece estadísticamente poco nos servía para reconocer los rostros, comentarios, miradas y emociones recogidas en sucesivas observaciones etnográficas. No nos resultó complicado diferenciar entre los jubilados que tenían en esta visita una actividad más, curiosa si se quiere, dentro de un tour por la vecina sierra de Aracena; escolares de la comarca que en visita casi obligada al museo confirmaban muchas de las lecciones y leyendas locales con las que iban creciendo; un grupo de vecinos del pueblo que acompañaban a familiares venidos de fuera y que mostraban orgullosos la importancia de la minería en la zona; antiguos trabajadores de la mina o riotinteños emigrados, que nos hacían percatarnos, al transitar las salas del museo, cómo se hacen llamativos determinados acentos catalanes, vascos o castellanos. Si uno entra en casual conversación o simplemente pone atención indiscreta a las conversaciones que estos visitantes mantienen, es usual escuchar cosas del tipo: "mi padre me contaba...", "mi madre me decía...". En una comarca de tan marcada tradición migratoria la figura del visitante que busca sus orígenes está siendo cada vez más usual y la del abuelo que intenta explicar al nieto en qué consiste la minería, también. El museo, y el parque minero en general, constituyen parte de estos "encuentros" que casi todos persiguen. $\mathrm{Al}$ emocional contraste con una calle, una casa, una dirección referida obsesivamente ("yo me crié en el número 30 de la calle Méndez Núñez"), la plaza de uno de los pueblos ("los domingos siempre íbamos al triángulo"), un bar ("en un baile de fin de año en el casino conocí a tu padre"), un comercio ("mis primeros pantalones largos me lo compraron en casa de..."), una atmósfera mil veces recreada, etc. El museo y su tratamiento científico de la memoria, contrapone una visión y un contexto en los que evocar a través de fotografías, utensilios, objetos, nombres propios, términos de la jerga minera local 
(catite, rodo, malacate, contramina...) perdida en la memoria y ahora súbitamente rescatada... en definitiva de algún pretexto que cataliza el recuerdo. Y en ese momento -registrado a partir de la observación participante- el visitante que busca en la comarca su pasado, el lugareño que evoca espacios tragados por la explotación minera, o el turista ajeno a la cultura minera que busca emociones exóticas, transmiten y comparten relatos, imaginaciones y recreaciones. El museo da soporte a memorias e ilusiones; será desde estas que los objetos patrimoniales adquieran sentidos personales que de nuevo podrán alejarse más o menos de los discursos expositivos oficializados en los textos y recorridos propuestos. El consumidor del patrimonio crea una experiencia propia al acercarse al objeto patrimonial.

El consumo del patrimonio, en su heterogeneidad, variabilidad y dinámica, supone el corazón de la patrimonialización (HernándezRamírez y Ruiz-Ballesteros 2005). Igual que en los procesos generales de la comunicación, en el campo del patrimonio hay que completar el momento, y sobre todo el contexto, de construcción o producción con el proceso de consumo o recepción. El objetivo no es otro que superar (complementándola) la perspectiva de estudio del patrimonio que se centra casi exclusivamente en la producción del mismo. Aun considerando el conflicto y la contestación, la perspectiva que se ocupa principalmente del momento de la producción de los bienes patrimoniales resulta incompleta porque cierra y limita el fenómeno patrimonial precisamente a las fases de la producción del mensaje, desconsiderando su recepción y el efecto de esa recepción, en definitiva su consumo tal como aquí lo abordamos desde una perspectiva comunicativa integral: con una mirada al contexto desde el que adquieren significado esos actos de consumo. Es por ello que entendemos que desde la perspectiva exclusiva de la producción del patrimonio solo cabe una descripción plana y excesivamente mecánica de la patrimonialización, porque si bien se asume el conflicto inherente a esos momentos de la producción del bien patrimonial, este se resuelve desde la consideración de unas relaciones de poder de corte hegemónico que presupone un modelo unidireccional de consumo.

La etnografía de diferentes procesos y momentos de la patrimonialización tanto en Riotinto como en Aguablanca (que van desde la declaración oficial de patrimonio, a la apertura de un museo, la visita y relatos de locales ante ese patrimonio, la reacción de visitantes, las resignificaciones de lugares y objetos, etc.), nos presenta, por el contrario, una respuesta altamente heterogénea, con un consumo que resulta ser coral y polifónico, donde las estrategias, elaboradas en, desde y para la producción del patrimonio por distintas instancias, tan solo son comprendidas cabalmente si atendemos a las prácticas de consumo que los sujetos locales despliegan en sus relaciones con él. Este es el más profundo sentido de la patrimonialización como proceso dinámico, abierto, sin fin.

En otro contexto bien diferente, Agua Blanca, el festival de la Balsa Manteña ilustra ese carácter siempre inacabado del patrimonio. Desde 1992 los manteños celebran este festival que rememora el primer encuentro de los indígenas costeños con los conquistadores en 1526. Surge para reivindicar "quinientos años de resistencia indígena" y en consonancia con el movimiento indigenista del país. Se celebra cada 12 de octubre. Ese día toda la comunidad en una espectacular comitiva, sale primero al municipio de Puerto López, y después a Machalilla (capitales del cantón y la parroquia, respectivamente, a la que pertenece Agua Blanca) para celebrar la comunidad del presente mediante su pasado y de su patrimonio. Así, en las inmediaciones del museo local, la mañana del doce de octubre unos treinta hombres adultos ataviados como "los antiguos manteños" comienzan a organizar la comitiva para el desfile. Entre ellos destacan el Gran Manta y el chamán, con atributos que les identifican: varas y objetos sagrados. Junto a ellos desfilan unos veinte niños igualmente ataviados, además de otro comunero que representa al Inca, con una imaginativa corona de aluminio y una capa azul. Los disfrazados son los componentes del comité arqueológico (el grupo encargado de la gestión turística del patrimonio local), que tienen la obligación -según se recoge en su reglamento interno-, bajo sanción, de participar en el desfile de la balsa manteña. Exhiben una indumentaria que compone una "tradición" con apenas quince años.

Al llegar a Puerto López comienza el desfile. El cortejo lo abre una pancarta, portada por jóvenes de la comunidad, con referencias al antiguo señorío manteño de Salangome del que Agua Blanca fue capital. Son seguidos por el resto de la comitiva, primero los niños y después los adultos que encarnan a indígenas rebeldes que corean gritos amenazadores 
“abajo los españoles"; “el pueblo unido jamás será vencido"; "viva Agua Blanca, capital del señorío de Salangome".

En el estruendo de tambores y consignas circula la chicha de maíz. Detrás de ellos un carro tira de la balsa manteña en la que van el Gran Manta y el chamán. De este modo se van recorriendo algunas de las principales calles de Puerto López, hasta llegar a la oficina del Parque Nacional Machalilla, donde se hace la primera parada. Normalmente el parque ofrece un refrigerio a los participantes e instala un equipo de megafonía para animar el acto. El chamán ofrece limpias a los asistentes, bajo la atenta mirada de curiosos y turistas, mientras el resto de participantes disfruta del refrigerio.

Posteriormente se toman los vehículos para viajar a Machalilla. Allí por fin la balsa y la comitiva llegan hasta la playa, al borde del océano Pacífico. Los indígenas manteños escenifican entonces el ritual central de la fiesta: una ceremonia de agradecimiento que dirige el Gran Manta, ayudado por el chamán. Un conjunto de agradecimientos y rogativas a las siete direcciones (norte, sur, este, oeste, arriba, abajo y al corazón), escenificados con emotividad. Un ritual que acaba ya sin turistas, interpretado exclusivamente para los habitantes de Machalilla y Agua Blanca. Mientras el chamán continúa haciendo limpias a pie de playa, el resto de asistentes comienzan la comida, el baile y la fiesta.

Esta fiesta les hace sentirse, y ser, manteños, al tiempo que cómplices en el proceso de indigenización. Con esta celebración exponen una forma de posesión del territorio, de fortalecer vínculos con el exterior, de relacionarse dentro de la comunidad, de conseguir proyectos y empleo, de aumentar el nivel de vida, de construirse y sentirse uno mismo en el medio en el que vive, desde el pasado hacia el futuro, incluso de sanarse. Lo manteño pareciera en definitiva una manera de guiar la acción de los hombres y mujeres de Agua Blanca. Este ritual pone en uso todos los elementos más destacados del patrimonio cultural de la comunidad: los restos y reminiscencias arqueológicas que han sido encontradas en su territorio y que se condensan en tanto en cuanto sirven para reivindicar la expresión indígena de la gente, que sin embargo ha sido muy recientemente reconocida, y resignificada. En el fondo un proceso de consumo análogo al que experimenta el exminero desarraigado cuando visita el museo minero de Riotinto.

\section{Sujetos y Consumo en los Procesos de Patrimonialización}

No hay proceso patrimonializador sin sujetos. Considerar la patrimonialización solo como un proceso estructural, cuasi-anónimo, de carácter institucional o producido por fuerzas globales (mercado, Estado) secuestra la posibilidad de acercarnos a la dimensión más social y humana que hay tras él. Los casos de Agua Blanca y Riotinto lo ilustran claramente. Es por ello que proponemos recuperar la noción de sujeto para estudiar el patrimonio: el sujeto como auténtico "consumidor" del patrimonio, como actor principal del proceso patrimonializador.

Hablamos de sujeto como consumidor de patrimonio en el sentido propuesto por Touraine (1993) cuando contextualiza la modernidad, insistiendo en que esta solo puede construirse en torno a la idea de sujeto y que este es el resultado del proceso de integración de las ambiciones de objetividad y subjetividad. Esta consideración que define al sujeto por su doble relación, a la vez de complementariedad y de oposición, es la que le otorga un papel determinante en los procesos de patrimonialización y en la producción social de significados en nuestros días, y la que hemos elegido para poder explicar la relación de los usuarios con el patrimonio.

Un patrimonio que en Riotinto o en Agua Blanca surge en la encrucijada de las dos grandes tendencias que nos ayudan a comprender el mundo en que vivimos: homogeneización económica y heterogeneización cultural. Profundamente asociado al sector turístico (en la vertiente económica), así como vinculado íntimamente a la generación de identidades (desde la perspectiva cultural). Sin embargo, por más que este papel del patrimonio esté claro a nivel macro, no acaba de entenderse cabalmente si no es constatado a nivel de los sujetos, inserto en esa perspectiva procesual que aquí reivindicamos. Solo así hemos sido capaces de comprender las similitudes de la patrimonialización en contextos tan dispares como la comunidad de Agua Blanca en Ecuador y Riotinto en Andalucía.

Por eso atender a los sujetos protagonistas del patrimonio insertos en los procesos de patrimonialización, nos ha ayudado a identificar: (1) la combinación de factores que reproducen mecanismos similares en cualquier parte del globo, eso que Ortiz (1998) caracteriza como globalización 
en relación con la economía y la técnica; y (2) aquellos otros factores que sirven para practicar y reivindicar la especificidad de universos culturales, potenciando los valores de la mundialización (Ortiz 1998). Encontramos comuneros de Agua Blanca que -por medio de la patrimonialización de restos arqueológicos y ecosistemas peculiares- sustentan una actividad turística que los legitima para ocupar un territorio protegido y asimismo para expresar su diferencia como pueblo indígena ancestral. Y en Riotinto mineros cuyas minas abandonadas, una vez convertidas en patrimonio, son vendidas como atracción turística al tiempo que procuran sostener, vía memoria e identidad, una autoestima puesta en cuestión. Dos realidades diversas y convergentes a un tiempo.

Llegar al nivel de los sujetos nos permite evidenciar que el patrimonio y los procesos de patrimonialización componen una manera imaginativa de estar en el mundo: "Entendida como un espacio de disputas y negociaciones simbólicas mediante el que los individuos y los grupos buscan anexar lo global a sus prácticas de lo moderno" (Appadauri 2001:8).

El patrimonio evidencia plenamente esa complejidad de la sociedad-mundo (Ortiz 1998). Las formas en las que se componen los procesos del patrimonio (desde su producción hasta su consumo) son una manera de hacer esa sociedad-mundo y por ende su estudio constituye una estrategia certera para comprender nuestras maneras de estar, como sujetos y como grupos, en ella. Aunque tratados de una manera muy sucinta, los casos de Agua Banca y Riotinto nos ayudan a comprenderlo.

Por eso nos interesa el análisis del proceso patrimonializador como hecho social y humano específico, señalando el papel del patrimonio como activador-creador de cultura, como proyección de futuro y no solo como reflejo del pasado-presente.

Una de las claves para comprender la dimensión integral del patrimonio es asumir que supone un acto de consumo por parte de los sujetos y que ese consumo es multidimensional y polifónico. Somos conscientes de que esta noción de consumo que proponemos para abordar el estudio del patrimonio requiere explicación. En nuestra sociedad actual es habitual reducir el concepto y uso del término "consumo" a aspectos exclusivamente económicos, vinculados irrevocablemente a transacciones monetarias y al omnipresente mercado. Para comprender el consumo patrimonial vamos más allá.
Las formas de apropiación, rechazo, indiferencia, o pasión respecto de los bienes patrimoniales que hemos encontrado en nuestros estudios, nos animan a emplear conscientemente esta acepción de consumo como forma de expresar de manera más amplia $-\mathrm{y}$ si se quiere provocadora- la relación entre sujetos y objetos. Patrimonialización no es solo comprar un recuerdo, vender la entrada a un museo, o vestirse con llamativos trajes prehispánicos para atraer turistas. El resultado de lo que acaba siendo patrimonio no es solo cuestión de qué, cómo o de quién sea el objeto patrimonializado, sino de las vinculaciones (diversas, históricas y complejas) que este objeto produce con cada uno de los sujetos con los que entra en contacto, a modo de acto comunicativo.

Para comprender qué hace la gente con el patrimonio y el patrimonio con la gente es fundamental atender a este acto de recepción, resaltando cómo estos receptores (consumidores) son, a su vez, productores del mismo proceso de comunicación en el que participan (Fuenzalida 1985; Lull 1997; Martin Barbero 1987; Orozco 1990). Si los procesos de patrimonialización nos han situado ante los sujetos, las teorías de la recepción nos permiten considerarlos agentes de dicho proceso. Eso es lo que se activa en el museo de Riotinto o en el festival de la Balsa Manteña.

Proponemos una noción de consumo que no está en contradicción con el mercado, entendido como un espacio para el encuentro de sujetos, situaciones, prácticas e ideologías diferenciadas. Así, consideramos el mercado, al igual que hace Ortiz (1998), como un contexto de socialización, "al lado de la familia, la religión y las naciones, modela la personalidad de los hombres (...). El espacio del mercado y del consumo se tornan así lugares en los cuales se engendran y comparten, patrones de cultura" (Ortiz 1998:101). El consumo así entendido nos presenta sujetos contextualizados, lo que nos ayuda a encarar las formas en las que estos participan en/ desde los procesos de patrimonialización tanto en el mercado como en diferentes contextos culturales.

Con grata sorpresa nos hemos encontrado tanto en Riotinto como en Agua Blanca con que las personas se relacionan con el patrimonio de formas bien distintas a las que marcan los discursos patrimonializadores oficialistas. Si bien es indudable la influencia que estos discursos hegemónicos producen en la patrimonialización y por tanto en los objetos seleccionados y en las configuraciones/ significaciones que estos toman, no lo es menos que 
la gente no es afectada de manera pasiva por ello, que las personas que consumen patrimonio traducen esos discursos en múltiples vertientes y con muy distintas reacciones. Nada mejor que un método como la etnografía para comprobarlo.

De esta forma, el consumo -como marco de la relación sujeto-patrimonio- constituye un hecho activo y comunicacional; no es el típico acto de consumo pasivo, sino que sirve al individuo, ante todo, para pensar y actuar. El consumo nos sitúa con sentido tanto en el mundo material como en el social, porque "cuando seleccionamos los bienes y nos apropiamos de ellos, definimos lo que consideramos públicamente valioso. Las maneras en que nos integramos y nos distinguimos en la sociedad" (García Canclini 1995:15). Así, las formas de apropiación y uso del patrimonio (consumo patrimonial) se muestran como verdaderos mediadores para conocer y situar a los sujetos, al tiempo que convierten al patrimonio en acción más que en objeto, al evidenciarlo como puro acto de consumo. Esta es la razón por la que insistimos en la potencialidad del consumo como perspectiva para encarar el estudio y análisis del patrimonio en toda su complejidad. No en vano es la estrategia analítica que nos permite entender qué ha hecho la gente con su patrimonio en Agua Blanca, dándole un uso que va desde lo económico a lo identitario sin contradicción aparente; o al efecto de la patrimonialización de la minería en Riotinto capaz de contribuir a restablecer la autoestima de los mineros al tiempo que es usado también para justificar las políticas públicas de reactivación económica.

Pero además, esta perspectiva que presenta al patrimonio como mediador -en la medida que desvela cómo se articula la relación de la gente con los objetos y con otros sujetos- saca a la superficie la dimensión política que posee todo proceso relacional y, por tanto, patrimonial. Como aquí lo hemos entendido, la patrimonialización nos enseña algo más que mecanismos de la microfísica del poder (en el sentido de disciplina, control y manipulación de Foucault (1986)), ya que deja espacio a la voluntad subjetiva, a la memoria particular, a las formas específicas y contextuales de ser y actuar, permitiendo de algún modo la resistencia táctica que contrarresta el interés estratégico (de Certeau 2000). Lo que hacen los mineros con el patrimonio (recuperando incluso el sentido de la lucha sindical) o los aguablanquenses al reivindicarse con su mediación como pueblo indígena ancestral, constituyen usos que contestan ampliamente al diseño patrimonial hegemónico.

El patrimonio es hoy un elemento básico en la construcción del sujeto social. Por eso es objeto de interés en la medida en que simboliza una forma de relación con el mundo, una selección de elementos de este para facilitar su lectura y trazar las oportunas relaciones afectivas. Patrimonializar nuestro mundo no es más que establecer una forma de vivirlo, habitarlo, sentirlo, con los inherentes conflictos que todo ello desata.

Los casos analizados nos muestran la multidimensionalidad del consumo patrimonial, situándonos en la encrucijada homogeneidadheterogeneidad que marca la sociedad-mundo (Ortiz 1998), combinando distintas dimensiones (económica, simbólica, sociopolítica, identitaria, de ocio y disfrute) que nos componen como sujetos sociales. En este sentido, y desde esta perspectiva, el patrimonio se convierte en un elemento homogeneizador-heterogeneizador a un tiempo, conjugando en sí el carácter más íntimo de la globalización: todos vivimos alrededor del patrimonio, pero los patrimonios son diferentes y particularmente vividos. El patrimonio nos iguala y nos diferencia.

Entendemos que mirar desde el consumo nos permite hacer confluir apropiación cultural $y$ relaciones de mercado sin tener que optar por una de las dos perspectivas para encauzar, sin contradicciones, nuestro análisis del patrimonio. De ahí nuestra propuesta de profundizar la idea de patrimonio como proceso, desde la perspectiva del consumo (recepción) integrada a la de construcción (producción), como estrategia para encarar en toda su complejidad el patrimonio: su multidimensionalidad, su carácter ambivalente y sobre todo su papel catalizador en nuestras maneras de hacer sociedad y constituirnos como sujetos en ella.

Agradecimientos: este artículo se ha elaborado en el contexto del proyecto de investigación "Retóricas de la naturaleza y turismo de base local: estrategias de sostenibilidad" (CSO2012-33044) financiado por el MINECO, España. Agradecemos de manera especial las recomendaciones de los revisores del texto. 


\section{Referencias Citadas}

Alsayyad, N. (ed.) 2001. Consuming Tradition, Manufacturing Heritage: Global norms and urban forms in the age of tourism. Routledge, London.

Angelo, D. 2014. Abriendo el debate en torno a patrimonio en Chungara. Chungara Revista de Antropología Chilena 46:73-176.

Appadauri, A. 2001. La Modernidad Desbordada. Dimensiones Culturales de la Globalización. Ed. Trilce y Fondo de Cultura Económica, Buenos Aires.

Ariño, A. 2002. La expansión del Patrimonio Cultural. Revista de Occidente 250:129-150.

Arrieta, I. 2009. Activaciones Patrimoniales e Iniciativas Museísticas: ¿Por Quién? ¿Para Qué? Universidad del País Vasco, Bilbao.

De Certeau, M. 2000. La Invención de lo Cotidiano. Artes de Hacer. Universidad Iberoamericana, México, D.F.

Foucault, M. 1986. Vigilary Castigar. Siglo XXI Editores, Madrid.

Fuenzalida, V. 1985. La recepción activa de televisión. Chasqui. Revista Latinoamericana de Comunicación 16:4-8.

García Canclini, N. 1995. Consumidores y Ciudadanos. Conflictos Multiculturales de la Globalización. Grijalbo, México, D.F.

Hernández-Ramírez, M. y E. Ruiz-Ballesteros 2005. Apropiación patrimonial en contextos mineros de Andalucía. Revista de Dialectología y Tradiciones Populares 60:103-127.

Hernández-Ramírez, M. y E. Ruiz-Ballesteros 2006. Intervenciones sobre el patrimonio minero en Andalucía: Análisis de los procesos de patrimonialización. En Anuario Etnológico de Andalucía, Consejería de Cultura, pp. 241-254. Junta de Andalucía, Sevilla.

Hernández-Ramírez, M. y E. Ruiz-Ballesteros 2008. El patrimonio como proceso social. Intervención, desarrollo y consumo del patrimonio minero en Andalucía. En Participación Ciudadana, Patrimonio Cultural y Museos: entre la Teoría y la Praxis, editado por I. Arrieta, pp. 129-148. Servicio editorial Universidad del País Vasco, Donostia.

Hernández-Ramírez, M. y E. Ruiz-Ballesteros 2011. Etnogénesis como práctica. Arqueología y turismo en el pueblo Manta (Ecuador). AIBR. Revista de Antropología Iberoamericana 6:159-191.

Iglesias, I. y E. Ruiz-Ballesteros1999. La conformación del patrimonio minero en Riotinto. En Cultura Minera en Andalucía, coordinado por E. Ruiz-Ballesteros. Demófilo Revista de Cultura Tradicional 32:9-36.

Lull, J. 1997. Medios, Comunicación y Cultura. Aproximación Global, Amorrortu editores, Buenos Aires/Madrid.

Martin Barbero, J. 1987. De los Medios a las Mediaciones. Comunicación, Cultura y Hegemonía. Gustavo Gili, Barcelona.

McEwan, C., M.I. Silva y C. Hudson 2006. Using the past to forge the future. The genesis of the community site museum at Agua Blanca, Ecuador. En Archaeological Site Museums in Latin America, editado por H. Silverman, pp. 187-216. University Press of Florida, Gainsville.

Martínez-Villarreal, R. y G. Oconitrillo 2012. Comunidades en la investigación de su pasado: herencia precolombina e historia oral en la comunidad de San Vicente de Nicoya, Costa Rica. Chungara Revista de Antropología Chilena 44:455-466.

Orozco, G. 1990. Notas metodológicas para abordar las mediaciones en el proceso de recepción televisiva. Diálogos de la Comunicación 2:54-63. FELAFACS, México, D.F.

Ortiz, R. 1998. Otro Territorio. Convenio Andrés Bello, Bogotá.

Prat, LL. 2006. La mercantilización del patrimonio: entre la economía turística y las representaciones identitarias. PH Boletín 58:72-80.

Pereiro, X. 2003. Patrimonialização e transformação das identidades culturais. En Portugal Chao, coordinadores J. Portela y J. Castro, pp. 231-247. Celta Editora, Oeiras.

Quintero, V. 2005. El patrimonio intangible como instrumento para la diversidad cultural ¿una alternativa posible? En Patrimonio Cultural, Multiculturalismo y Gestión de la Diversidad, editado por G. Dietz y G. Carrera, pp.15-29. Cuaderno del IAPH. Consejería de Cultura, Sevilla.

Quintero, V. 2009. Los Sentidos del Patrimonio. Alianzas y Conflictos en la Construcción del Patrimonio Etnológico Andaluz. Fundación Blas Infante, Sevilla.

Romero, E., E. Ruiz, J.M. Pérez, E. Aguilera y R. Aguilera 2003. Informe BIC sobre la Cuenca minera de Riotinto para su declaración como sitio Histórico. Boletín PH. Instituto Andaluz del Patrimonio Histórico 45:43-50.

Rosas, A.1999. La participación social en las nuevas políticas para el Patrimonio Cultural. En Patrimonio Etnológico. Nuevas Perspectivas de Estudio, coordinado por E. Aguilar Criado, pp. 34-51. Junta de Andalucía, Instituto Andaluz del Patrimonio Histórico, Granada.

Ruiz-Ballesteros, E. (coord.) 1999a. Cultura Minera en Andalucía. Demófilo Revista de Cultura Tradicional.

Ruiz-Ballesteros, E. 1999b. Intervenciones sobre el patrimonio minero en Riotinto: de la identificación colectiva al recurso económico. En Patrimonio Cultural y Museología, coordinado por J. Agudo y E Fernández, pp. 111-118. FAAES, Santiago de Compostela.

Ruiz-Ballesteros, E. 2001a. Minería, Cultura y patrimonio en Andalucía. En Antropología de Andalucía, dirigido por S. Rodriguez, pp. 354-382. Hércules de ediciones, La Coruña.

Ruiz-Ballesteros, E. 2001b. Cultura, medio ambiente y minería. En Minería y Medio Ambiente. Perspectiva Histórica, coordinado por J.C. Pérez Cebada, pp. 27-47. Universidad de Huelva, Huelva.

Ruiz-Ballesteros, E. 2009. Agua Blanca. Comunidad y Turismo en el Pacífico Ecuatorial. Abya-Yala, Quito.

Ruiz-Ballesteros, E. y M.A. Gual-Font 2012. The emergence of new commons. Community and multilevel governance in the Ecuadorian coast. Human Ecology 40:847-862.

Ruiz-Ballesteros, E. y M. Hernández-Ramírez 2007. Identity and community. Reflections on the development of mining heritage tourism in Southern Spain. Tourism Management 28:677-687.

Ruiz-Ballesteros, E. y M. Hernández-Ramírez 2010. Tourism that empowers? Commodification and appropriation in Ecuador's turismo comunitario. Critique of Anthropology 30:201-229. 
Ruiz-Ballesteros, E., M. Hernández y E.M. Fedriani Martel 2008. The development of mining heritage tourism: A systemic approach. En Tourism Development: Economics, Management and Strategy, editado por A.D. Ramos y P. Jimenez, pp. 121-143. Nova Science Publishers, Nueva York.

Ruiz-Ballesteros, E. y E. Rubio de Miguel 2008. Lo natural y lo contaminado: ironías del río Tinto. En Patrimonialización de la Naturaleza. El Marco Social de las Políticas Ambientales, coordinado por O. Beltrán, J. Pascual e I. Vaccaro, pp. 165-180. Ankulegi Antropologia Elkartea, Donosti.

Ruiz-Ballesteros, E., J.M. Valcuende, V. Quintero, J.A. Cortes y E. Rubio 2009. Naturalizing the environtment, Perceptual frames, senses and resitance. Journal of Material Culture 14:147-167.

Touraine, A. 1993. Crítica a la Modernidad. Temas de Hoy, Madrid. 
ANDROS IMPRESORES

wWw.androsimpresores.cl 

